\title{
TILING THREE-DIMENSIONAL SPACE WITH POLYHEDRAL TILES OF A GIVEN ISOMORPHISM TYPE
}

\author{
BRANKO GRÜNBAUM, PETER MANI-LEVITSKA AND G. C. SHEPHARD
}

\section{Introduction}

In this paper we give a partial answer to a question posed by Ludwig Danzer at the London Mathematical Society Symposium 'Relations between finite and infinite dimensional convexity' held in Durham in July 1975. Let $P$ be any convex polyhedron, that is, a compact set in $E^{3}$ which is the intersection of a finite number of closed half-spaces. Then Danzer asked whether it is possible to find a face-to-face tiling of $E^{3}$ by polyhedra each of which is combinatorially isomorphic to $P$. In this generality the question is still open; here we shall establish an affirmative answer for the case in which $P$ is simplicial, that is, all its two-dimensional faces are triangles.

Our result may be regarded as a three-dimensional analogue of the fact, first observed by Schlegel (1883) [6] that for each $n \geqslant 3$, the plane can be tiled (in an edge-to-edge manner) by convex $n$-gons. (Of course, if $n \geqslant 7$ then such a tiling is necessarily non-normal, see Grünbaum and Shephard [4]. However in this paper we shall not consider questions concerning normality of tilings. For a brief account of such problems in the three-dimensional case, see Danzer, Grünbaum and Shephard (1983) [2], and for further information concerning tilings by congruent tiles in $E^{3}$, see Grünbaum and Shephard (1980) [3]. Note that in Russian literature the word 'normal' is used with a different meaning, namely to indicate the property we call 'face-to-face'.)

In order to make this paper self-contained, the first section will contain a summary of the necessary definitions. This will be followed by a statement of the main theorem, and subsequent sections will be devoted to its proof. Finally we shall make some general comments and state some open problems.

\section{Definitions and main theorem}

Let $P$ be a convex polyhedron in $E^{3}$ and let $E$ be a supporting plane of $P$. If the set $E \cap P$ is two-dimensional it is called a face of $P$, and such a face is necessarily a convex polygon. If $E \cap P$ is one-dimensional it is called an edge of $P$, and if $E \cap P$ is of dimension zero then it is called a vertex of $P$. If all the faces of $P$ are triangles, then $P$ is a simplicial polyhedron.

A tiling (tessellation) of $E^{3}$ is a family $\mathscr{T}=\left\{T_{i}: i=1,2, \ldots\right\}$ of sets (the tiles of $\mathscr{T})$ such that the union of the tiles $\bigcup_{i \geqslant 1} T_{i}$ is $E^{3}$ and no two distinct tiles $T_{i}$ have common interior points. The tiling $\mathscr{T}$ is said to be convex if each $T_{i}$ is a convex set, and is locally finite if every compact set in $E^{3}$ meets only a finite number of tiles. If $\mathscr{T}$ is convex and locally finite, and the tiles $T_{i}$ are compact, then it is easy to see that

Received 3 December, 1982.

The research of the first author was supported by N.S.F. Grant No. MCS-8001570 and by a Fellowship from the Guggenheim Foundation. 
each tile is a convex polyhedron. Such a tiling is called face-to-face if the intersection of every pair of distinct tiles is either empty or is a face, an edge or a vertex of each. In this case the intersection of every family of tiles, if not empty, is necessarily a face, an edge or a vertex of every tile in the family.

Two polyhedra $P_{1}, P_{2}$ are said to be isomorphic (or combinatorially equivalent) if there exists an inclusion preserving bijection between the sets of faces, edges and vertices of $P_{1}$ and the set of faces, edges and vertices of $P_{2}$. For a given convex polyhedron $P$, a tiling $\mathscr{T}$ is called monotypic of type $P$ if each of the tiles of $\mathscr{T}$ is a convex polyhedron isomorphic to $P$. (The word monotypic has been used in a completely different sense in McMullen, Schneider and Shephard (1974) [5]; however we do not believe that this is likely to cause any confusion.)

We can now state our main theorem which confirms, for simplicial polyhedra, a result which we suspect may be true for all convex polyhedra.

MaIN THeOREM. Let $P$ be a (compact three-dimensional) convex simplicial polyhedron. Then there exists a locally-finite face-to-face convex tiling $\mathscr{T}$ which is monotypic of type $P$.

For brevity, in the sequel, the word 'tiling' will be used without further qualification to mean a locally-finite face-to-face convex tiling.

We shall now give a sketch of the proof of the theorem and explain some of the difficulties that arise in establishing a result of this nature. Let $P$ be the given convex simplicial polyhedron. Then for $k=1,2, \ldots$ we construct a 'core' $\mathscr{T}_{k}=\left\{T_{i}: 1 \leqslant i \leqslant n_{k}\right\}$ consisting of $n_{k}$ isomorphic copies of $P$ with disjoint interiors. To construct $\mathscr{T}_{k+1}$ from $\mathscr{T}_{k}$ we adjoin $n_{k+1}-n_{k}$ further isomorphic copies of $P$, suitably chosen so that

$$
B_{k}=\bigcup_{1 \leqslant i \leqslant n_{k}} T_{i}=\operatorname{set} \mathscr{T}_{k}
$$

is, for each $k$, star-shaped with respect to some fixed point $O$ and $B_{k}$ contains a sphere $K_{k}$, centred at $O$, whose diameter increases without bound as $k \rightarrow \infty$. Then the 'direct union' $\bigcup_{k \geqslant 1} \mathscr{T}_{k}$ is clearly a tiling of the kind required in the proof of the
theorem.

As will be seen, the fundamental difficulty is in choosing the isomorphic copies of $P$ so that, as each successive core is constructed, the sets $B_{k}$ have the required properties. To this end we shall state and prove in the next section a lemma describing certain 'distortions' or transformations of a convex polyhedron which do not alter its combinatorial type, but enable one to prescribe the shape of the transformed polyhedron in the neighbourhood of one of its vertices. It is clear that such transformations play a fundamental role in the combinatorial theory of polytopes; it is therefore strange that we have been unable to find any results of this nature in the literature. In fact it seems that authors who wished to transform a convex polyhedron into one of the same combinatorial type have, almost without exception, used only projective and affine transformations. For the proof of our theorem such transformations are not sufficient. A description of the most general transformation of a convex polyhedron which preserves combinatorial type seems, at present, to be completely unattainable.

If $F$ is any face of a convex polyhedron $P$ it will be found convenient to define a Schlegel point $X$ of $P$ with respect to the face $F$ as any point such that the joins of $X$ 
to all the vertices of $P$ other than those that belong to $F$ meet the plane of $F$ in relatively interior points of $F$. It is easy to see that the set of all Schlegel points of $P$ with respect to $F$ (or Schlegel set as we shall call it) is an open convex set-in fact it is the intersection of a finite number of open half-spaces whose boundaries are the planes of $F$ and of the faces of $P$ which are incident with $F$.

We conclude this section by summarising some of the properties of (nonsingular) affine transformations which we shall use in our proofs. Any affine transformation $\Phi$ applied to a convex polyhedron preserves convexity and combinatorial type, and also maps Schlegel points into Schlegel points. Such a transformation $\Phi$ is determined uniquely by the images of any four non-coplanar points. In particular if $\Phi$ maps the non-coplanar points $A, B, C$ and $D$ into $A, B, C$ and $E$ respectively, then the whole of the plane $\pi$ containing $A, B$ and $C$ is pointwise invariant under $\Phi$. Moreover, if the point $D$ is nearer to the plane $\pi$ than is the point $E$, then $\Phi$ will increase all distances from $\pi$; more precisely, for any point $X$ the distances of $X$ and $\Phi(X)$ from $\pi$ will be in the same ratio as are the distances of $D$ and $E$ from $\pi$.

\section{A lemma about polyhedra}

Let $P$ be a convex simplicial polyhedron with a $j$-valent vertex $V(j \geqslant 3)$, let $Q$ be a $j$-gonal (convex) pyramid, and let $\lambda \geqslant 1$ be a real number. Then we shall say that $P$ is $\lambda$-adaptable to $Q$ at $V$ if there exists a convex polyhedron $P^{\prime}$ which is combinatorially isomorphic to $P$ and has the following three properties.

(i) If $V^{\prime}$ is the vertex of $P^{\prime}$ which corresponds to the vertex $V$ of $P$ in the isomorphism (or in one chosen isomorphism if there exists more than one), then the $j$ vertices of $P^{\prime}$ which are joined by edges of $P^{\prime}$ to $V^{\prime}$ are all coplanar. We shall use $\pi$ to denote the plane containing these $j$ vertices.

(ii) The intersection of $P^{\prime}$ with the closed half-space that is bounded by $\pi$, and which contains $V^{\prime}$, is a $j$-gonal pyramid directly congruent to the given pyramid $Q$; further, in this congruence, $V$ corresponds to the apex of $Q$.

(iii) The distances from $V^{\prime}$ of all the vertices of $P^{\prime}$ (other than $V^{\prime}$ and those lying in $\pi$ ) is at least $\lambda$ times the distance of $\pi$ from $V^{\prime}$.

In other words, $P$ is $\lambda$-adaptable to $Q$ at $V$ if the pyramid $Q$ can be 'continued' in such a way as to arrive at a polyhedron $P^{\prime}$ which is of the same combinatorial type as $P$ and whose vertices are 'far' from $V$. The convex polyhedron $P^{\prime}$ will be referred to as a $\lambda$-adaptation of $P$ to $Q$ at $V$.

Lemma. Let $P$ be a convex simplicial polyhedron. Then for some $j$ satisfying $3 \leqslant j \leqslant 5$ there exists a j-valent vertex $V$ of $P$ such that, for every $j$-gonal convex pyramid $Q$, and for every $\lambda \geqslant 1, P$ is $\lambda$-adaptable to $Q$ at $V$.

In Figure 1 we show a convex simplicial polyhedron $P$ with eight vertices obtained from an octahedron (with vertices $A B C D E F$ ) by erecting two 'low' pyramids, one with apex $G$ on the face $A C D$, and one with apex $H$ on the face $A B E$. It is clear that the vertex $B$ of $P$ cannot play the role of the vertex $V$ of the lemma since the existence of the edge $A E$ of $P$ precludes the coplanarity of the vertices which correspond to $A, C, E, F$ and $H$ in any polyhedron $P^{\prime}$ isomorphic to $P$. For similar 


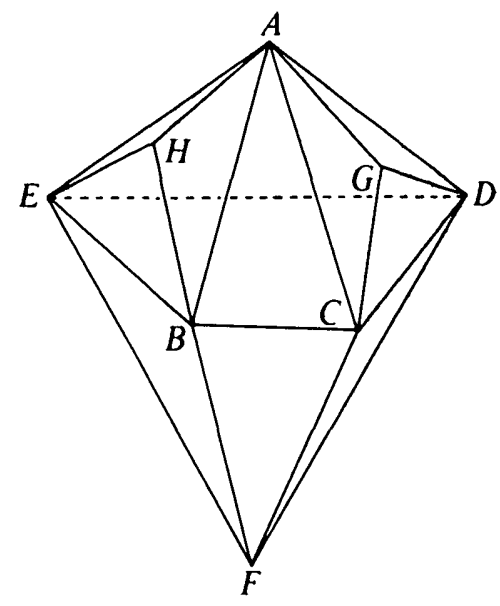

FIG. 1

reasons, each of the vertices $A, C, D$ and $E$ is also unsuitable. On the other hand, any of the vertices $F, G$ or $H$ may be chosen as $V$ and then the lemma can be established. It is still necessary, of course, to show that the other conditions for $\lambda$-adaptability can be fulfilled; for example, if $F$ is chosen as the vertex $V$ then we must show how to construct $P^{\prime}$ in such a way that the quadrangle corresponding to $B C D E$ is congruent to the base of the given quadrangular pyramid $Q$.

We shall begin our proof of the lemma by explaining how the vertex $V$ is to be chosen. If $P$ possesses a 3 -valent vertex then that vertex may be chosen as $V$. In this case the lemma is almost trivial since the tetrahedron defined by $V$ and the three vertices joined to $V$ by edges of $P$ can be transformed by an affine transformation into one which is congruent to any given triangular pyramid (tetrahedron) $Q$.

If $P$ does not possess a 3 -valent vertex, then the choice of $V$ is more difficult, and we proceed in the following manner. Suppose that $L, M, N$ are vertices of the polyhedron $P$ such that $L M, M N$ and $N L$ are edges of $P$, but $L M N$ is not a face of $P$, then we shall refer to the edge circuit $L M N$ as a missing face of $P$. For example $A B E$ and $A C D$ are missing faces of the polyhedron shown in Figure 1. If a simplicial polyhedron $P$ has no missing faces then we say it is fully faced. Thus a fully faced (simplicial) polyhedron $P$ is one such that every edge circuit of length 3 is the boundary of some face of $P$. If $P$ is not fully faced and $L M N$ is a missing face, it can be cut by the plane containing $L M N$ into two polyhedra $P_{1}$ and $P_{2}$ each of which is simplicial-and in this case we shall refer to the faces $L M N$ (of $P_{1}$ and of $P_{2}$ ) as faces of attachment. By repeating this process of cutting by planes defined by missing faces, it is clear that $P$ can be cut up into a finite set of components, each of which is a fullyfaced simplicial polyhedron. Since this decomposition of $P$ has a 'tree-like' structure, at least one component has at most one face of attachment. (In fact if $P$ has no missing faces then there is just one component, and if $P$ has at least one missing face, then there will be at least two components each with one face of attachment.)

Write $P^{*}$ for any (fully-faced) component of $P$ with one face of attachment $L M N$. It is a well-known consequence of Euler's theorem for convex polyhedra (see, for example, Grünbaum and Shephard [4]) that if $v_{i}$ denotes the number of 
$i$-valent vertices of a simplicial polyhedron, then

$$
3 v_{3}+2 v_{4}+v_{5}=12+\sum_{i \geqslant 7}(i-6) v_{i} \geqslant 12
$$

from which it follows that $P^{*}$ must have at least four vertices of valence strictly less than 6 . Hence we may select $V$ as a vertex (of valence 3,4 or 5) of $P^{*}$ which is distinct from $L, M$ and $N$. Further, the subgraph of $P^{*}$ induced by the vertices of $P^{*}$ adjacent to $V$-that is the set of edges of $P^{*}$ for which both end-points are joined to $V$ by edges of $P^{*}$ - is a simple circuit. That it has no diagonals is a consequence of the fact that $P^{*}$ is fully faced.

We now forget about $P^{*}$ and think of $V$ as a vertex of the original polyhedron $P$. In the case where $P$ is fully faced we may select any vertex of $P$ of valence 3,4 or 5 . The remainder of the proof of the lemma consists in verifying that the required conditions can be met, with $V$ selected as just described.

Let $\mathscr{G}$ be the graph consisting of all the vertices of $P$ except $V$, and all the edges of $P$ except for the $j$ edges $(j=3,4$ or 5 ) incident with $V$. From the above remarks we see that the subgraph of $\mathscr{G}$, determined by the $j$ vertices of $P$ which are joined to $V$ by edges, is a simple circuit of length $j$. Let us denote it by $C(j)$. Further, except in the trivial case where $j=3$ and $P$ is a tetrahedron, $\mathscr{G}$ is 3 -connected and planar. Therefore, by the strengthening of Steinitz' theorem established in Barnette and Grünbaum (1970) [1], $\mathscr{G}$ can be realised as the graph of edges and vertices of a convex polyhedron $\bar{P}$ with any prescribed convex $j$-gon $F(j)$ as the face of $\bar{P}$ whose boundary corresponds to the circuit $C(j)$. In particular, $F(j)$ can be chosen to be congruent to the base of the $j$-sided pyramid $Q$ of the lemma.

Suppose that $Q$ is situated so that its base coincides with $F(j)$. Let $U$ be a Schlegel point of $\bar{P}$ with respect to $F(j)$ and define $\Phi$ to be the non-singular affine transformation which leaves every point in the plane of $F(j)$ fixed, but maps $U$ onto the apex of $Q$. Then it is clear that $Q \cup \Phi(\vec{P})$ is a convex polyhedron isomorphic to $P$.

Moreover, since $U$ is any Schlegel point of $\bar{P}$ with respect to $F(j)$ we may choose it arbitrarily close to $F(j)$. Then $\Phi$ will map the vertices of $\bar{P}$ (other than those of $F(j))$ into points which are arbitrarily far from the plane of $F(j)$. In particular, for any $\lambda \geqslant 1$, all the vertices of $\Phi(\bar{P})$ (other than those of $F(j)$ ) can be made to lie at distances greater than $\lambda h$ from the apex of the pyramid $Q$, where $h$ is the height of $Q$. Then $P^{\prime}=Q \cup \Phi(\bar{P})$ satisfies all the conditions of the lemma-it is a $\lambda$-adaptation of $P$ to $Q$ at $V$, the apex of $Q$.

\section{Proof of the main theorem}

In Section 2 we gave a brief outline of the proof of the main theorem, and we shall continue with the notation and terminology introduced there; we use $\mathscr{T}_{k}$ $(k=1,2, \ldots)$ for the sequence of cores, and write $B_{k}=\bigcup_{1 \leqslant i \leqslant n_{k}} T_{i}$. Our construction will ensure that each $B_{k}$ is star-shaped from some fixed point $O$, and $B_{k} \subset B_{k+1}$ for each $k$. The construction begins with $B_{1}=\mathscr{T}_{1}=P$, and $O$ as any interior point of $P$. Let us suppose that we have defined $\mathscr{T}_{k}$ for some $k \geqslant 1$; we now describe in detail how to construct $\mathscr{T}_{k+1}$ by adjoining to $\mathscr{T}_{k}$ tiles $T_{i}\left(n_{k}<i \leqslant n_{k+1}\right)$, each of which is an isomorphic copy of $P$. The difficulty is to do this in such a way that $B_{k+1}$ is starshaped, so that the construction may be continued. To avoid trivialities we shall 
assume throughout that $P$ is not a tetrahedron for in this case the theorem is trivially true.

Because $B_{k}$ is star-shaped, its boundary is a triangulated topological sphere-the triangulation being induced by the faces, edges and vertices of the tiles $T_{i}$ in $\mathscr{T}_{k}$ that lie on the boundary of $B_{k}$. Let $X$ be a vertex of $B_{k}$ that lies nearest to $O$, and let $Y$ be the point on the ray $O X$ such that $X$ is the mid-point of $O Y$. Write $\mathscr{X}_{s}$ for the star of $X$ in the boundary of $B_{k}$, that is, the triangulated disk consisting of all the triangular faces of $B_{k}$ which have $X$ as a vertex. If we write $t$ for the number of such triangles, they may be conveniently denoted by $X A_{m} A_{m+1}$ for $m=1,2, \ldots, t$, where each $A_{i}$ is a vertex of $B_{k}$ and $A_{t+1}=A_{1}$. In Figure 2 we show a polyhedron $P$ with $t=5$; the five triangles of $\mathscr{X}_{s}$ are shaded. It will be observed that all the triangles of $\mathscr{X}_{s}$ are visible from $Y$.

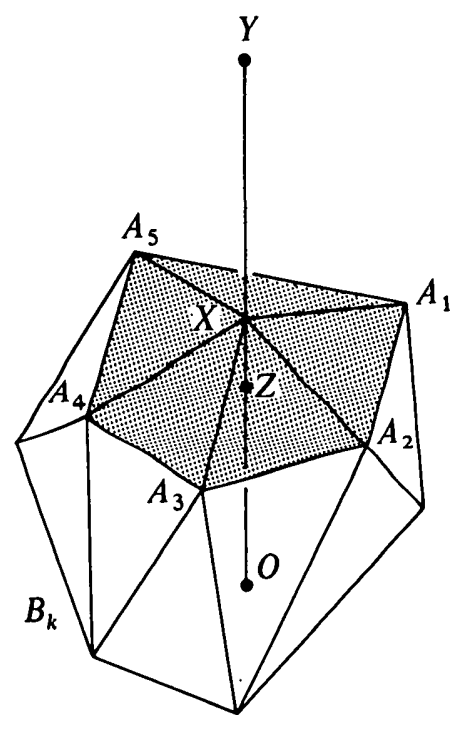

FIG. 2

We now select a $j$-valent vertex $V$ of $P(j=3,4$ or 5$)$ as in the proof of the lemma of Section 3. The construction which follows depends on the value of $j$ and we need to discuss the three cases separately.

(a) If $j=3$ then for $m=1,2, \ldots, t$ we use $Q_{m}$ to denote the triangular pyramid (tetrahedron) with base $Y A_{m} A_{m+1}$ and apex $X$. By the lemma we can, for each value of $m$ and each $\lambda_{m} \geqslant 1$, construct a $\lambda_{m}$-adaptation $P_{m}^{\prime}$ of $P$ to $Q_{m}$ at $X$. For each $m$ choose $\lambda_{m}$ so large that every vertex of $P_{m}^{\prime}$ (other than $X, A_{m}$ and $A_{m+1}$ ) is at least twice as far as $X$ from the point $O$. Then the polyhedra $P_{1}^{\prime}, \ldots, P_{t}^{\prime}$, together with the polyhedra $P_{1}, \ldots, P_{n_{k}}$ that belong to the core $\mathscr{T}_{k}$, completely cover a neighbourhood of $X$ : see Figure 3. However, we cannot take this set of polyhedra as the next core since, in general, their union will not be star-shaped from $O$. Hence we must apply a further modification in the following way.

For each $m$ let $\bar{P}_{m}$ be the convex polyhedron which is the closure of $P_{m}^{\prime} \backslash Q_{m}$. From the proof of the lemma we see that $X$ is a Schlegel point for $\bar{P}_{m}$ with respect to the face $Y A_{m} A_{m+1}$. Since every Schlegel set is open, we can find a point $Z_{m}$, close to $X$ and lying in the relative interior of the line segment $O X$, which is also a Schlegel point for $\bar{P}_{m}$. Further, if we choose $Z$ in the relative interior of the segment $O X$ and at 


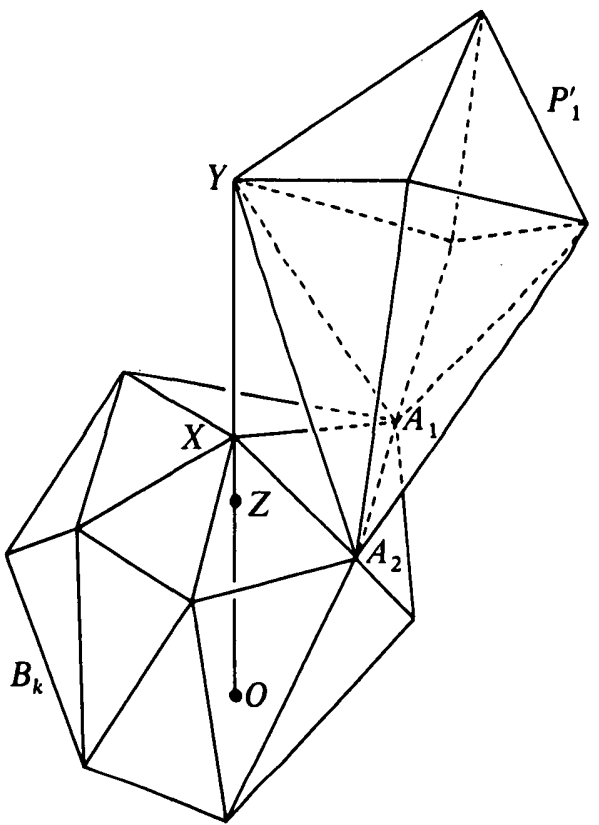

FIG. 3

least as close to $X$ as any of the $t$ points $Z_{m}$, then $Z$ will be a Schlegel point for each of the polyhedra $\bar{P}_{1}, \ldots, \bar{P}_{t}$. For each $m$ let $\Phi_{m}$ be that affine transformation which leaves the plane $X A_{m} A_{m+1}$ pointwise invariant and maps $Z$ onto $O$. Let $n_{k+1}=n_{k}+t$ and define $P_{n_{k}+m}=\Phi_{m}\left(P_{m}^{\prime}\right)$ for $m=1,2, \ldots, n_{k+1}-n_{k}=t$. Then the core $\mathscr{T}_{k+1}=\left\{P_{1}, \ldots, P_{n_{k+1}}\right\}$ will have the required properties; it is made up of polyhedra isomorphic to $P$ fitting together in a face-to-face manner, and the union of these polyhedra $B_{k+1}$ is star-shaped from $O$. Moreover, from the method of construction and the fact that each $\Phi_{m}$ tends to increase lengths, all the 'new' vertices of $B_{k+1}$ (that is to say, those that are not also vertices of $B_{k}$ ) are at least twice as far from $O$ as is the vertex $X$. The latter condition implies that as $k \rightarrow \infty$, the largest spheres $K_{k}$ contained in $B_{k}$ have diameters which increase without bound.

This completes the proof of the theorem in the case $j=3$.

(b) If $j=5$, the proof requires one intermediate step. We write $R$ for the convex polyhedron with Schlegel diagram shown in Figure 4. It is bounded by fifteen pentagons and three triangles, and can be written as the union of fifteen pentagonal pyramids, each of which has the vertex marked $V$ as apex, and has one of the fifteen pentagonal faces as its base.

We now proceed as in the case $j=3$ up to the point where we defined $P_{1}^{\prime}, \ldots, P_{1}^{\prime}$, except that we use the new polyhedron $R$ instead of $P$. Let us denote the polyhedra so obtained by $R_{1}^{\prime}, \ldots, R_{t}^{\prime}$; we note that the union of these $t$ polyhedra can be written as the union of $15 t$ pentagonal pyramids each with apex $X$. Let us denote these pentagonal pyramids by $Q_{1}, \ldots, Q_{15 t}$. Applying the lemma again, we define, for $i=1, \ldots, 15 t$, a polyhedron $P_{i}^{\prime \prime}$ which is a $\lambda_{i}$-adaptation of $P$ to $Q_{i}$ at $X$. As before, each $\lambda_{i}$ is to be chosen so that all the vertices of each $P_{i}^{\prime \prime}$ (other than $X, A_{1}, \ldots, A_{t}$ ) are at least twice as far from $O$ as the point $X$.

Write $\bar{P}_{i}$ for the closure of $P_{i}^{\prime \prime} \backslash Q_{i}(i=1, \ldots, 15 t)$ and construct $Z$ in the relative interior of the line segment $O X$ (close to $X$ ) so that $Z$ is a Schlegel point for each of 


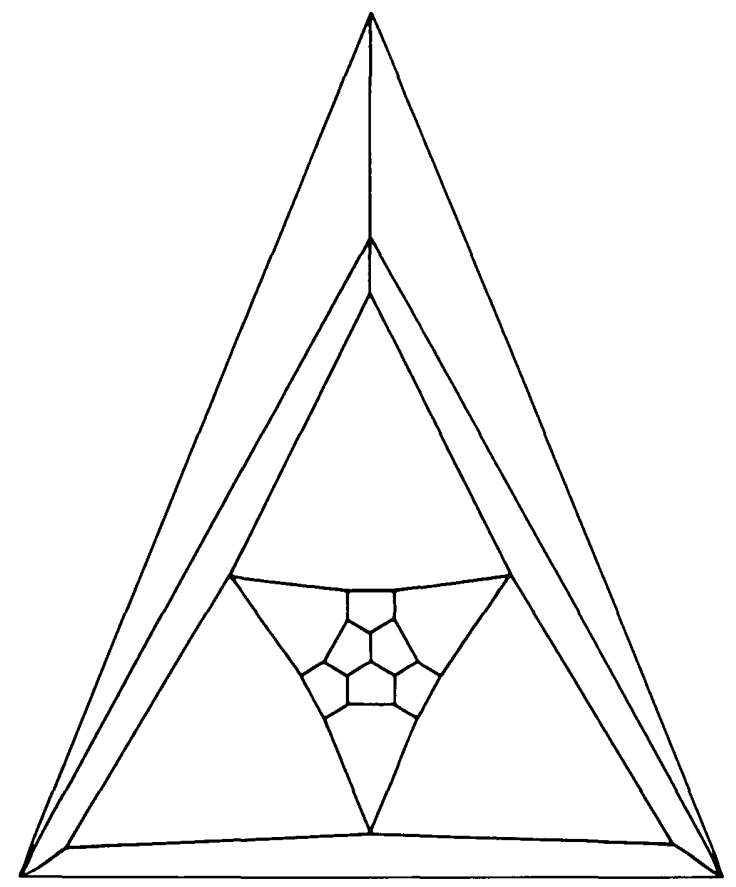

FIG. 4

the $\bar{P}_{i}$ relative to its pentagonal face. If the pentagonal pyramid $Q_{i}$ is part of $R_{m}^{\prime}$ then we apply to $P_{i}^{\prime \prime}$ an affine transformation $\Phi_{m}$ which maps $Z$ onto $O$ and leaves the plane $X A_{m} A_{m+1}$ pointwise invariant. Write $n_{k+1}=n_{k}+15 t$ and define $T_{n_{k}+i}=\Phi_{m}\left(P_{i}^{\prime \prime}\right)$ for $i=1,2, \ldots, n_{k+1}-n_{k}=15 t$. For reasons similar to those described in the case $j=3$, the core $\mathscr{T}_{k+1}=\left\{T_{1}, \ldots, T_{n_{k+1}}\right\}$ will have the required properties; it is made up of polyhedra isomorphic to $P$ which fit together in a face-to-face manner, and the union $B_{k+1}$ of these polyhedra is star-shaped. Further, spheres $K_{k} \subset B_{k}$ will have diameters which increase without bound as $k \rightarrow \infty$. The theorem is therefore proved in the case $j=5$ also.

(c) If $j=4$ the construction is slightly more complicated. The underlying reason for this is that there exists no convex polyhedron of which three faces are triangles and all the remaining faces are quadrangles-if such a polyhedron existed we could use it in the same way as we used the polyhedron $R$ in the case $j=5$. Because of this, instead of dealing with each of the triangles $X A_{m} A_{m+1}$ separately as we did in the previous cases, it is necessary to take them in pairs. We must therefore distinguish between the cases where their number $t$ is even or is odd.

We begin with the case where $t$ is odd. If every vertex of $P$ has even valence then it is clear that the same will be true of every star-shaped set built up (face-to-face) from isomorphic copies of $P$ in any manner whatsoever. Thus if $t$ is odd it follows that $P$ has a vertex of odd valence-let us denote this vertex by $W$. Then choose an affine transformation $\Psi$ with the following four properties:

(i) $\Psi(W)=X$;

(ii) $\Psi$ maps one of the triangular faces $F$ of $P$ which is incident with $W$ onto one of the triangular faces of $B_{k}$ which is incident with $X$;

(iii) $O$ is a Schlegel point of $\Psi(P)$ with respect to the face $\Psi(F)$; 
(iv) all the vertices of $\Psi(P)$ other than those belonging to $\Psi(F)$ are at least twice as far from $O$ as is the point $X$.

The effect of adjoining $\Psi(P)$ to $\mathscr{T}_{k}$ is to change the valence of $X$ (as a vertex of $B_{k}$ ) from odd to even. Hence we have reduced the case of odd $t$ to that of even $t$, and so we need only consider the latter possibility.

Let $X, A_{1}, \ldots, A_{t}$ be defined as before, with $t=2 r$. For $s=1, \ldots, r$ let $C_{s}$ be a point on the ray from $X$ to the mid-point of the line segment with end-points $Y, A_{2 s}$, let $D_{s}$ be a point on the ray from $X$ to the centroid of the triangle $Y A_{2 s-1} A_{2 s}$, and let $E_{s}$ be a point on the ray from $X$ to the centroid of the triangle $Y A_{2 s} A_{2 s+1}$. These points $C_{s}, D_{s}$ and $E_{s}$ must be chosen so that the following conditions hold.

(i) The distance from $O$ to each of these points must be at least twice that of $X$ from 0 .

(ii) $X, Y, A_{2 s-1}, A_{2 s}, C_{s}, D_{s}$ are the vertices of a convex polyhedron $R_{s}$ with six triangular faces and one quadrangular face $X Y C_{s} A_{2 s}$.

(iii) $X, Y, A_{2 s}, A_{2 s+1}, C_{s}, E_{s}$ are the vertices of a convex polyhedron $S_{s}$ with six triangular faces and one quadrangular face that coincides with that of $\boldsymbol{R}_{s}$.

Now truncate $R_{s}$ at the 4-valent vertex $D_{s}$ to obtain a convex polyhedron $R_{s}^{\prime}$ with two triangular and six quadrangular faces, and, in a similar manner, truncate $S_{s}$ at the vertex $E_{s}$ to obtain a polyhedron $S_{s}^{\prime}$ of the same combinatorial type as $R_{s}^{\prime}$. The construction is illustrated in Figure 5 which shows all the edges of $R_{s}^{\prime}$ and $S_{s}^{\prime}$ other than those incident with $X$. It will be observed that $R_{s}^{\prime} \cap S_{s}^{\prime}$ can be expressed as the union of ten quadrangular pyramids each with apex $X$ and with one of the quadrangular faces of $R_{s}^{\prime}$ or $S_{s}^{\prime}$ as base (namely the ten quadrangles shown in Figure $5)$. Let these pyramids be denoted by $Q_{1}, \ldots, Q_{5 t}$.

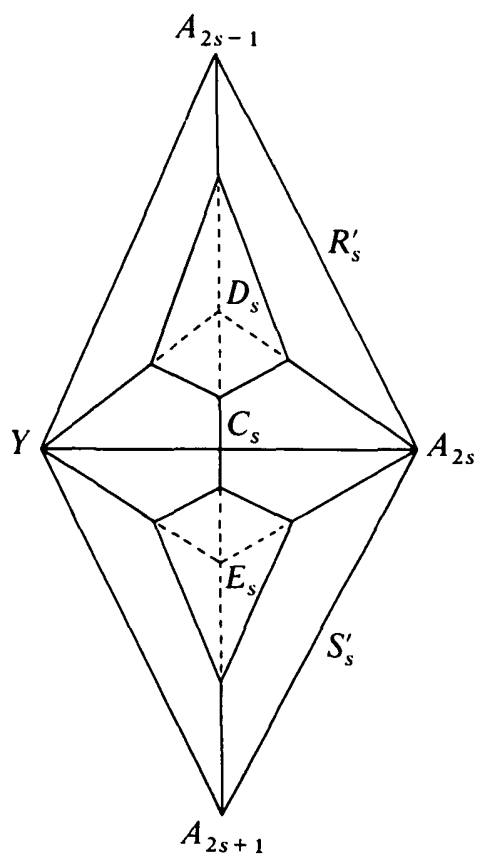

FIG. 5 
We now proceed exactly as in the case $t=5$. We define, for each $i$, a $\lambda_{i}$-adaptation $P_{i}^{\prime \prime}$ of $P$ to $Q_{i}$ at $X$, with $\lambda_{i}$ large enough for all the vertices of $P_{i}^{\prime \prime}$ other than those belonging to $Q_{i}$ to be at distances from $O$ at least twice that of $X$. Finally we define affine transformations $\Phi_{m}$, as before, to ensure that the new core $\mathscr{T}_{k+1}=\left\{T_{1}, \ldots, T_{n_{n+1}}\right\}$ with $T_{n_{k}+i}=\Phi_{m}\left(P_{i}^{\prime \prime}\right)\left(i=1, \ldots, 5 t=n_{k+1}-n_{k}\right)$ leads to a starshaped set $B_{k+1}$.

The only point that needs clarification is to show that the resulting tiles $T_{i}$ fit together face-to-face. The construction ensures that all the polyhedra $P_{i}^{\prime \prime}$ are face-toface, and we simply have to check that the same is true of their images under the affine transformations $\Phi_{m}$. This follows because, for every even value of $m$, the restrictions of $\Phi_{m}$ and of $\Phi_{m-1}$ to the plane containing $X, Y$ and $A_{m}$ coincide. Thus $\Phi\left(C_{m}\right)=\Phi_{m-1}\left(C_{m}\right)$ and the assertion follows.

Thus the theorem is true for $j=4$ also, and this completes the proof in all cases.

The argument for $j=4$ applies, of course, to the case where $P$ is an $n$-gonal bipyramid. It is worth noting that the isomorphic copies of $P$ can be chosen, in this case, so that the equatorial $n$-gon is plane. Hence each bipyramid can be cut into two pyramids and the theorem then implies that a tiling by convex $n$-gonal pyramids is possible for all $n \geqslant 3$.

\section{Remarks and open problems}

The outstanding problem in this area is, of course, the one originally posed by Ludwig Danzer. Does the main theorem remain true if the word 'simplicial' is deleted from the statement? We conjecture that this is so, though any proof would require much more powerful methods than those described here. In fact, until a great deal more is known about transformations of polyhedra that preserve isomorphism type, any attempt at proof seems doomed to failure. The same remark applies to generalisations to higher dimensions, and we can see little prospect of proving any results of any kind except in the most elementary case-where the $n$-dimensional simplicial polytope $P$ has a vertex of valence $n$. It is worth noting that even in threedimensional space the theorem cannot be extended to unbounded polyhedral sets $P$ since the latter may have all vertices with valences at least six. Possibly some results may be salvaged in this case if we restrict the valences of the vertices, for example, by insisting that $P$ is a simple set (all vertices of valence 3 ).

As stated in the introduction, in this paper we have not been concerned with questions of normality. We mention, however, that as proved in Danzer, Grünbaum and Shephard (1983) [2], there exist polyhedra, even simplicial ones, for which no normal monotypic face-to-face tiling of $E^{3}$ is possible. This raises the interesting question of trying to characterise polyhedra which admit such tilings.

For every $p \geqslant 3$ there exists an edge-to-edge tiling of the plane by $p$-gons with every vertex of valence $q$ if $p^{-1}+q^{-1} \leqslant \frac{1}{2}$. Such a tiling is uniquely determined within a homomorphism of the plane by the integers $p$ and $q$, and moreover, each tiling is topologically tile-transitive, edge-transitive and vertex-transitive. It even possesses a realisation in either the Euclidean or hyperbolic plane which is regular. The question we wish to raise is whether any analogous results hold in the threedimensional case, either in general, or for particular choices of $P$ as the isomorphism type of the tile. It is clear that the constructions described in this paper do not, in 
general, lead to tilings with any transitivity properties, and results of this nature, if true, would require constructions of a substantially different nature. Again it seems that any results along these lines would be extremely difficult to establish.

\section{References}

1. D. Barnette and B. Grunbaum, 'Preassigning the shape of a face', Pacific J. Math., 32 (1970), 299-306.

2. L. Danzer, B. Grunbaum and G. C. Shephard, 'Does every type of polyhedron tile three-space?' Structural Topology, 8 (1983), 3-14.

3. B. Grunbaum and G. C. Shephard, 'Tilings with congruent tiles', Bull. Amer. Math. Soc., 3 (1980), 951-973.

4. B. Grunbaum and G. C. ShePhard, Tilings and patterns (Freeman, San Francisco), to appear.

5. P. MCMullen, R. SChNeider and G. C. Shephard, 'Monotypic polytopes and their intersection properties', Geom. Dedicata, 3 (1974), 99-129.

6. V. SCHLEGEL, 'Theorie der homogen zusammengesetzten Raumbebilde', Nova Acta Leopoldina, 44 (1883), 337-459, and plates xiv-xxii.
Department of Mathematics, University of Washington, Seattle,
Washington 98195 , U.S.A.

\author{
Mathematisches Institut, \\ Universität Bern, \\ Sidlerstrasse 5 , \\ CH-3000 Bern, \\ Switzerland.
}

\footnotetext{
University of East Anglia, Norwich NR4 7TJ.
} 